\title{
Influencia de las condiciones edáficas sobre la dominancia y diversidad de árboles en bosques pluviales tropicales del Chocó biogeográfico
}

\author{
Harley Quinto-Mosquera ${ }^{1}$, Darlin Hurtado ${ }^{2} \&$ Julay Arboleda ${ }^{1}$ \\ 1. Programa de Biología, Facultad de Ciencias Básicas. Universidad Tecnológica del Chocó Diego Luis Córdoba, \\ Quibdó, Chocó, Colombia; hquintom@gmail.com, Julay_015@hotmail.com \\ 2. Grupo de Investigación en Ecología y Conservación de Ecosistemas Tropicales, Facultad de Ciencias Básicas, \\ Universidad Tecnológica del Chocó Diego Luis Córdoba, Quibdó, Chocó, Colombia; dhmsidai@gmail.com
}

\author{
Recibido 30-V-2019. Corregido 25-VII-2019. Aceptado 24-IX-2019.
}

\begin{abstract}
Influence of edaphic conditions on the dominance and diversity of trees in tropical rainforests of the biogeographic Chocó. Introduction: The biogeographic Chocó presents an exuberant diversity of arboreal species; however, it is unknown if soils determine this diversity. Objective: Evaluate how edaphic conditions explain the diversity and arboreal dominance in tropical pluvial forests at a local scale. Methods: Seven permanent research plots were established in the forests of Opogodó, Pacurita and Salero. In these plots, tree diversity and dominance were measured, and edaphic conditions such as organic matter (OM), acidity, nutrients and texture were determined. Results: It was found that the diversity was much greater in Salero and Pacurita; but the dominance was greater in Opogodó. The diversity was positively related to phosphorus, aluminum, silt and clay; while, with $\mathrm{pH}, \mathrm{OM}$, calcium, magnesium, potassium and sand, the relationship was negative. On the other hand, dominance was negatively related to $\mathrm{P}$ and $\mathrm{Al}$; while, the relationship was positive with $\mathrm{pH}, \mathrm{OM}, \mathrm{Ca}$ and sand. Conclusions: It was corroborated that the arboreal diversity is negatively related to some variables $(\mathrm{Ca}, \mathrm{Mg}, \mathrm{K})$ of edaphic fertility. Apparently, edaphic infertility, acidity and aluminum toxicity, determine tree dynamics and diversity.
\end{abstract}

Key words: aluminum, edaphic fertility, Opogodó, Pacurita, phosphorus, Salero, species richness.

Quinto-Mosquera, H., Hurtado, D. \& Arboleda, J. (2019). Influencia de las condiciones edáficas sobre la dominancia y diversidad de árboles en bosques pluviales tropicales del Chocó biogeográfico. Revista de Biología Tropical, 67(6), 1278-1291.

Los bosques lluviosos tropicales de América latina, poseen una gran diversidad de especies arbóreas (Gentry, 1988; Phillips, Hall, Gentry, Sawyer, \& Vizquez, 1994; Valencia, Balslev, Paz, \& Mino, 1994), y son considerados como los ecosistemas terrestres más biodiversos del planeta (Hartshorn, 2002; Myers, Mittermeier, Mittermeier, Da Fonseca, \& Kent, 2000). Dentro de los cuales, se han registrado zonas con más de 300 especies por hectárea (Gentry, 1988; Valencia et al., 1994). Los factores que explican esta alta diversidad arbórea han sido de gran interés. Particularmente, Leigh et al. (2004), en una revisión de literatura consideraron que esta alta diversidad se encuentra explicada por factores como la limitación en dispersión, heterogeneidad de hábitats, suelos, topografía, disturbios, presión por patógenos, productividad, clima, y tasas de especiación y extinción basados en modelos neutrales y filtros ambientales. Asimismo Antonelli y Sanmartin (2011) consideraron que los factores que explican la alta diversidad de árboles en el Neotrópico se pueden clasificar en 
dos categorías: los factores bióticos (adaptaciones edáficas, interacciones con polinizadores, dispersores y herbívoros, nicho y limitación en dispersión), y los abióticos (tiempo, precipitación, temperatura, área geográfica, topografía e hidrología). Dichos factores explican en distintas proporciones la diversidad arbórea (Givnish, 1999).

Las condiciones edáficas han sido consideradas como un factor ambiental que explica significativamente la alta diversidad de especies arbóreas en bosques lluviosos tropicales (Huston, 1980; Gentry, 1981; Clark, Clark, \& Read, 1998; Clark, 2002; Quinto \& Moreno, 2014; Nadeau \& Sullivan, 2015). Por ejemplo, Huston (1980, 1994) sintetizó varios trabajos que proponen que la diversidad de plantas es baja en suelos pobres en nutrientes, aumenta conforme incrementa el nivel de nutrientes, y finalmente vuelve a disminuir cuando el suelo es muy fértil. En consecuencia, se ha planteado la hipótesis de que en condiciones extremas de baja y/o alta fertilidad edáfica, la diversidad arbórea tropical tiende a disminuir (Clark, 2002; Huston, 1980, 1994, 2012).

Con el objetivo de poner a prueba dicha hipótesis, Huston (2012) estudió la relación existente entre la precipitación, la fertilidad del suelo, la productividad primaria neta y la diversidad arbórea de diferentes regiones del mundo. En los sitios con mayor precipitación, la productividad primaria neta y la fertilidad edáfica tienden a disminuir, pero la diversidad de especies es alta (Huston, 2012). Asimismo, Huston (1980) evaluó la riqueza de especies arbóreas y su relación con los nutrientes del suelo en boques lluviosos tropicales de Costa Rica y encontró que la diversidad tiende a disminuir con la fertilidad del suelo (sumatoria de los porcentajes de $\mathrm{P}, \mathrm{K}$ y Ca). En suelos pobres en nutrientes ( $\mathrm{P}, \mathrm{K}$ y $\mathrm{Ca}$ ) se registra la mayor riqueza de especies arbóreas (Huston, 1980; Nadeau \& Sullivan, 2015). Esta tendencia en diversidad arbórea también ha sido reportada por Faber-Langendoen y Gentry (1991), Clinebell, Phillips, Gentry, Stark, y Zuuring (1995), Duque, Cavelier, y Posada (2003), y Quinto y Moreno (2014), quienes han encontrado altos valores de riqueza de especies arbóreas en suelos pobres en nutrientes de diferentes regiones tropicales. Particularmente, Clinebell et al., (1995) reportaron correlaciones negativas de la riqueza de especies arbóreas con el contenido de $\mathrm{K}, \mathrm{Mg}$, Ca y fertilidad del suelo; mientras que, con el $\mathrm{Al}$ y $\mathrm{Fe}$ la relación tiende a ser positiva. Mientras que, Condit, Engelbrecht, Pino, Pérez, y Turner (2013), evidenciaron una asociación entre la composición y riqueza de especies arbóreas y el contenido de humedad de la estación seca, el $\mathrm{P}$ disponible y el Ca. Sin embargo, algunos trabajos muestran que el efecto de las variables edáficas sobre la diversidad y composición florística es poco significativo (Barreto, Duque, Cárdenas, \& Moreno, 2010; Duque et al., 2003; Quinto \& Moreno, 2014; Valencia et al., 2004).

El Chocó biogeográfico es una región del neotrópico ubicada al noroeste de Sudamérica, considerado como un hotspot de conservación debido a su alta biodiversidad y endemismo (Myers et al., 2000). Allí se han registrado entre 100 y 300 especies arbóreas por hectárea (Faber-Langendoen \& Gentry, 1991; Gentry, 1988; Quinto \& Moreno, 2014). Gentry (1988) atribuyó esta inusual diversidad a los altos niveles de precipitación y ausencia de sequías, aunque este supuesto ha sido poco contrastado, pues en la región se han realizado pocos estudios.

En un estudio realizado en la localidad de Salero (Chocó biogeográfico), se encontró que la riqueza de especies arbóreas se relaciona positivamente con el Al del suelo y negativamente con la micro-topografía (Quinto \& Moreno, 2014). Sin embargo, la escala de evaluación fue local, lo que posiblemente incidió en los resultados, porque no se evaluaron gradientes contrastables de condiciones edáficas. En consecuencia no se encontró evidencia que apoye la hipótesis de Huston $(1980,1994)$ y Clinebell et al., (1995) sobre diversidad arbórea y contenido de nutrientes del suelo en esta región. El objeto del presente estudio es evaluar como las condiciones edáficas (acidez, MO, $\mathrm{Al}, \mathrm{N}$ total, $\mathrm{P}$ disponible, $\mathrm{K}, \mathrm{Mg}, \mathrm{Ca}$, capacidad de intercambio catiónico, y textura) explican 
la diversidad y dominancia arbórea, a escala regional, empleando tres bosques pluviales tropicales ubicados en localidades independientes (Opogodó, Pacurita y Salero) en el Chocó biogeográfico. Para ello, se comparó la diversidad arbórea a nivel de unidades de muestreo (400 $\mathrm{m}^{2}$ ), y mediante curvas de diversidad se evaluó la acumulación de especies. Además, nos planteamos la hipótesis de que en suelos con altos contenidos de $\mathrm{Al}$, y pobres en nutrientes como el $\mathrm{N}$ total, $\mathrm{K}, \mathrm{Mg}, \mathrm{Ca}$, la riqueza de especies arbóreas podría ser alta. Asimismo, basados en Condit et al. (2013), se espera que el P disponible del suelo tenga una asociación positiva con la riqueza de especies arbóreas en bosques del Chocó biogeográfico.

\section{MATERIALES Y MÉTODOS}

Área de estudio: El estudio se realizó en los bosques lluviosos tropicales de las localidades de Pacurita (municipio de Quibdó), Salero (municipio de Unión Panamericana) y Opogodó (municipio de Condoto), departamento del Chocó, Colombia. Estas localidades hacen parte de la subregión ecogeográfica Central Norte del Chocó biogeográfico que comprende la totalidad de las cuencas altas de los ríos Atrato y San Juan. Estos bosques presentan una altura de entre 70 y 150 m.s.n.m. y poseen una precipitación promedio anual de 7 500-10 000 mm (Quinto \& Moreno, 2016). Los suelos son ultisoles, extremadamente ácidos, con alta saturación de $\mathrm{Al}$ y con muy bajas concentraciones de $\mathrm{P}, \mathrm{Mg}$, Ca y CICE. Los contenidos de K son intermedios. Mientras que la MO y el N Total son muy altos, especialmente en Opogodó. En términos de textura, los suelos de Pacurita presentan mayor contenido de arcilla, en Opogodó son arenosos, y en Salero poseen más limo (Quinto \& Moreno, 2016). La composición arbórea de estas localidades está dominada por especies como: Mabea chocoensis, Pouteria sp., Oenocarpus bataua, Eschweilera pittieri, Croton jorgei, Wettinia quinaria, Eschweilera aff. integrifolia, Brosimum utile, entre otras. También están presentes las familias Sapotaceae, Euphorbiaceae, Lecythidaceae,
Arecaceae, Myristicaceae, Vochysiaceae y Clusiaceae (García et al., 2003; Quinto \& Moreno, 2014).

Establecimiento de parcelas: En la localidad de Salero se emplearon dos parcelas permanentes de investigación establecidas entre abril y agosto de 1998 mediante la metodología BIOTROP (García et al., 2003), las cuales hacen parte de la red de parcelas permanentes de Colombia (Vallejo-Joyas et al., 2005). Cada una de estas parcelas consiste de un cuadrado de $500 \times 20$ m dividido en 25 cuadrantes de 20 x $20 \mathrm{~m}$, que se emplearon como unidades de muestreo de diversidad y dominancia arbórea. Por su parte, en las localidades de Opogodó y Pacurita, se emplearon parcelas de $100 \times 100 \mathrm{~m}$ instaladas en el 2013, divididas en 25 cuadrantes de 20 x 20 m. En Opogodó, se establecieron tres parcelas de una hectárea, mientras que en Pacurita se instalaron dos parcelas de una ha de bosque. En cada una de los cuadrantes de $20 \mathrm{x}$ $20 \mathrm{~m}$ se realizaron mediciones de los parámetros físicoquímicos del suelo (acidez, $\mathrm{MO}, \mathrm{Al}$, $\mathrm{N}$ total, $\mathrm{P}$ disponible, $\mathrm{K}, \mathrm{Mg}, \mathrm{Ca}$, capacidad de intercambio catiónico, y textura) que se detallan en Quinto y Moreno (2016).

Inventario de árboles en las parcelas: Se midió el diámetro a la altura del pecho (DAP) (1.3 m sobre el nivel del suelo) a todos los árboles con DAP $\geq 10 \mathrm{~cm}$. A todos los árboles medidos se les determinó el hábito de crecimiento en las categorías de árbol, bejuco o liana y palma, se anotaron las observaciones particulares de cada individuo.

Identificación botánica: Se identificaron todas los individuos hasta el máximo nivel taxonómico posible ( $N N$, especie, género, familia botánica) en el herbario de la Universidad Tecnológica del Chocó "D.L.C." "Herbario Chocó". Esta identificación se llevó a cabo utilizando las claves especializadas de Gentry (1993) y Mahecha (1997).

Análisis de datos: Los datos de los inventarios de los árboles de las parcelas se 
emplearon para determinar la abundancia de las especies, con lo cual se determinó la riqueza, diversidad y dominancia arbóreas en las parcelas y cuadrantes (Moreno, 2001). La diversidad alfa se determinó mediante los siguientes índices de diversidad: dominancia de Simpson y de Berger-Parker, equidad de Shannon-Wiener y diversidad de Margalef (Magurran, 1988; Moreno, 2001). Para hacer más robustas las evaluaciones de diversidad y dominancia arbórea, se emplearon los coeficientes o exponentes de Hill, para obtener la riqueza efectiva a partir de los índices de Shannon-Winner y Simpson (Hill, 1973; Moreno 2001, Jost, 2006). Específicamente, se determinó la serie de números de Hill (Hill, 1973; Moreno 2001), de siguiente forma:

$\mathrm{N} 0=$ número total de especies $(\mathrm{S})$

$\mathrm{N} 1=$ número de especies abundantes $=\mathrm{e}^{\mathrm{H}^{\prime}}$

$\mathrm{N} 2=$ número de especies muy abundantes = $1 / \lambda$

Donde H' es el índice de Shannon-Winner, $\lambda$ es el índice de Simpson. Igualmente, se determinó el índice de equidad de Hill (Hill, 1973), de la siguiente forma: $E^{\prime}=N 2 /$ $\mathrm{N1}$; donde $\mathrm{N} 2$ es el número de especies muy abundantes, y $\mathrm{N} 1$ es el número de especies abundantes (Hill, 1973; Moreno, 2001). Los valores de diversidad y dominancia arbórea de los cuadrantes se compararon mediante la prueba no paramétrica de Kruskal-Wallis, dado que no se cumplieron los supuestos de normalidad y homogeneidad de varianzas, evaluados con los estadísticos de Bartlett y Hartley (Hoshmand, 1998). Adicionalmente, se determinaron las curvas de acumulación de especies con los estimadores no paramétricos de diversidad y riqueza de especies, Jacknife 1, Chao 2 y Bootstrap (Chao, Chazdon, Colwell, \& Shen, 2005; Colwell, Mao, \& Chang, 2004). Estas curvas se determinaron con el programa EstimateS (Chao et al., 2005; Colwell et al., 2004, Colwell et al., 2012). La precisión de los estimadores se estimó evaluando el sesgo y exactitud por medio de la comparación de la riqueza estimada y la verdadera (López-Gómez
\& Williams-Linera 2006). Para este análisis se emplearon las fórmulas de Chiarucci, Enright, Perry, Miller, y Lamont (2003):

$$
\begin{gathered}
\text { Sesgo }=\left(\mathrm{S}_{\text {estimada }}-\mathrm{S}_{\text {verdadera }}\right) / \mathrm{S}_{\text {verdadera }} \\
\text { Exactitud }=\left(\left(\mathrm{S}_{\text {estimada }}-\mathrm{S}_{\text {verdadera }}\right) / \mathrm{S}_{\text {verdadera }}\right)^{2}
\end{gathered}
$$

Las correlaciones entre las variables de diversidad y las condiciones edáficas se evaluaron mediante el coeficiente de correlación por rangos de Spearman y el análisis de componentes principales (ACP). Para la construcción del ACP y la matriz de correlación se tomaron los datos de diversidad, riqueza, equidad, dominancia arbórea, y los valores de las variables edáficas (acidez, MO, Al, nutrientes, CICE, y textura) registradas en cada una de las unidades de muestreo $\left(400 \mathrm{~m}^{2}\right)$, y se efectuaron las respectivas correlaciones.

Finalmente, se emplearon regresiones lineales múltiples para evaluar la relación conjunta existente entre la riqueza, equidad de Shannon y dominancia de Simpson con las variables edáficas. Las variables edáficas fueron transformadas con $\log (+1)$ para reducir su anormalidad y las que fueron significativas en la regresión múltiple se seleccionaron con el método de Stepwise - Backward (gradualmente hacia atrás). En general, los análisis se realizaron con cuatro programas estadísticos, para comparar la diversidad entre unidades de muestreo se empleó el Statgraphics Centurion XV (Statistical Graphics Corp., 2002), los índices de diversidad se determinaron con el Past (Hammer, Harper, \& Ryan, 2001) las curvas de acumulación de especies se generaron con el EstimateS 9 (Chao et al., 2005; Colwell et al., 2004, 2012) y los análisis de componentes principales se realizaron con el CANOCO 4.5 (ter Braak \& Šmilauer, 2002).

\section{RESULTADOS}

En los bosques del Chocó biogeográfico se registró la mayor riqueza total de especies (sp) por hectárea (ha) en la localidad de Salero, con 219; en Opogodó menor riqueza total de especies, con 75 (Tabla 1). La mayor abundancia 
TABLA 1

Índices de diversidad alfa para las especies arbóreas de bosques tropicales del Chocó biogeográfico, Colombia

TABLE 1

Alpha diversity indices for tree species from tropical forests of the biogeographic Chocó, Colombia

\begin{tabular}{lccccccc}
\multicolumn{1}{c}{} & \multicolumn{2}{c}{ Salero } & \multicolumn{3}{c}{ Opogodó } & \multicolumn{2}{c}{ Pacurita } \\
\multicolumn{1}{c}{ Índices de diversidad alfa } & Parcela E & Parcela U & PI & PII & PIII & PIV & PV \\
Riqueza de especies (esp./ha) & 219 & 179 & 90 & 75 & 78 & 97 & 93 \\
Abundancia de individuos (ind./ha) & 712 & 644 & 955 & 708 & 636 & 913 & 979 \\
Dominancia de Simpson (D) & 0.01 & 0.02 & 0.027 & 0.032 & 0.032 & 0.027 & 0.03 \\
Equidad de Shannon-Weinner (H') & 4.81 & 4.63 & 3.91 & 3.76 & 3.77 & 3.97 & 3.89 \\
Diversidad de Margalef (I) & 33.19 & 27.52 & 12.97 & 11.28 & 11.93 & 14.09 & 13.36 \\
Dominancia de Berger-Parker (D) & 0.05 & 0.07 & 0.057 & 0.08 & 0.073 & 0.071 & 0.077 \\
N1 de Hill (número de especies abundantes) & \multicolumn{2}{c}{$20.57 \mathrm{a}$} & & $16.46 \mathrm{~b}$ & & $19.74 \mathrm{a}$ \\
N2 de Hill (número de especies muy abundantes) & \multicolumn{2}{c}{$18.74 \mathrm{a}$} & & $14.10 \mathrm{c}$ & & $16.83 \mathrm{~b}$ \\
Equidad de Hill & \multicolumn{2}{c}{$0.90 \mathrm{a}$} & & $0.85 \mathrm{~b}$ & & $0.85 \mathrm{~b}$ \\
\hline
\end{tabular}

de individuos se registró en Pacurita, con 979 árboles/ha; mientras que, en Opogodó se registró la menor abundancia con 636 árboles/ha (Tabla 1). La mayor dominancia de Simpson y de Berger-Parker se registró en Opogodó. Por su parte, la mayor equidad de ShannonWeinner y diversidad de Margalef se registró en Salero (Tabla 1). Los números de especies abundantes de Hill (N1) mostraron un mayor valor en las localidades de Salero y Pacurita, mientras que el número de especies muy abundantes de Hill (N2) y la equidad de Hill fueron mayores en los bosques de Salero (Tabla 1).

A nivel de cuadrantes, la riqueza fue similar en Pacurita y Salero con promedios de 22.96 y 22.16 especies/cuadrante, respectivamente. Mientras que, en Opogodó fue significativamente menor con 19.1 especies/cuadrante (K $2,172=23.22 \mathrm{P}<0.0001$ ) (Fig. 1). Contrario a ello, la dominancia de Simpson fue significativamente mayor en Opogodó (Fig. 1). Las curvas de acumulación de especies mostraron que en Salero se alcanzó la mayor acumulación de especies, con un máximo de 301 especies en dos ha muestreadas. En Opogodó se registró un total de 119 especies en tres ha. Asimismo en Pacurita se registró un total de 113 especies arbóreas en dos ha (Fig. 2). En ambas localidades (Opogodó y Pacurita), los estimadores de diversidad, Jack 1, Chao 2 y Bootstrap, presentaron valores cercanos a los
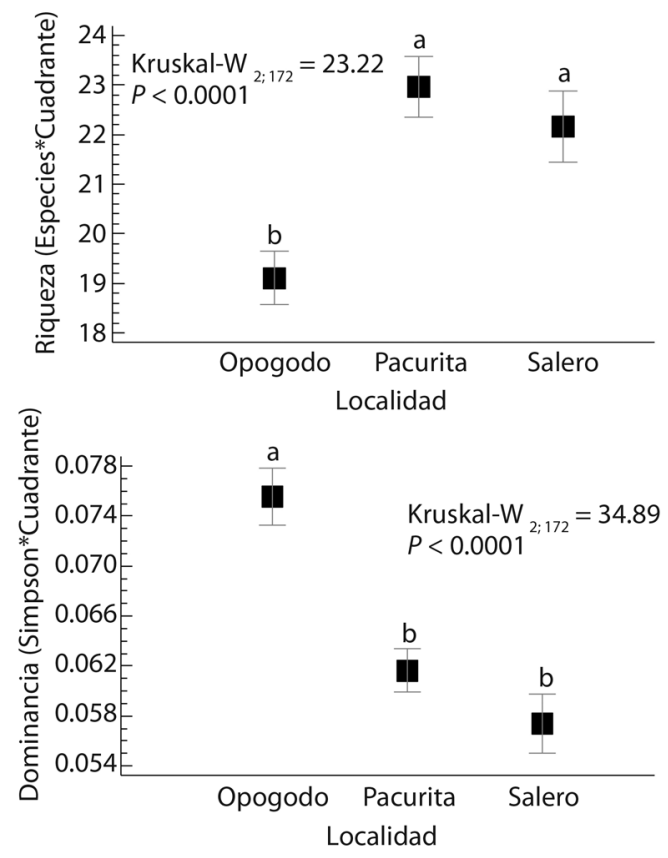

Fig. 1. Riqueza y dominancia de especies arbóreas de los bosques lluviosos tropicales de las localidades de Opogodó, Pacurita y Salero en el Chocó biogeográfico, Colombia.

Fig. 1. Richness and dominance of arboreal species of the tropical rainforests of the localities of Opogodó, Pacurita and Salero in the biogeographical Chocó, Colombia.

de la diversidad observada (Fig. 2). En consecuencia los valores de sesgos y exactitudes de los estimadores fueron muy bajos (Tabla 2). En 
TABLA 2

Comparación de estimadores de diversidad (Jacknife 1, Chao 2, Bootstrap), sesgos y exactitudes de tres bosques tropicales del Chocó biogeográfico, Colombia

TABLE 2

Comparison of diversity estimators (Jacknife 1, Chao 2, Bootstrap), biases and accuracies of three tropical forests of the biogeographic Chocó, Colombia

\begin{tabular}{lccc} 
Estimadores de diversidad & Salero & Opogodó & Pacurita \\
Especies totales observadas & 301 & 119 & 113 \\
Jacknife 1 & 429.38 & 139.72 & 136.52 \\
Chao 2 & 458.45 & 137.84 & 137.59 \\
Bootstrap & 357.26 & 128.90 & 123.41 \\
Sesgo de Jacknife 1 & 0.427 & 0.174 & 0.208 \\
Exactitud de Jacknife 1 & 0.182 & 0.030 & 0.043 \\
Sesgo de Chao 2 & 0.523 & 0.158 & 0.218 \\
Exactitud de Chao 2 & 0.274 & 0.025 & 0.047 \\
Sesgo de Bootstrap & 0.187 & 0.083 & 0.092 \\
Exactitud de Bootstrap & 0.035 & 0.007 & 0.008 \\
\hline
\end{tabular}
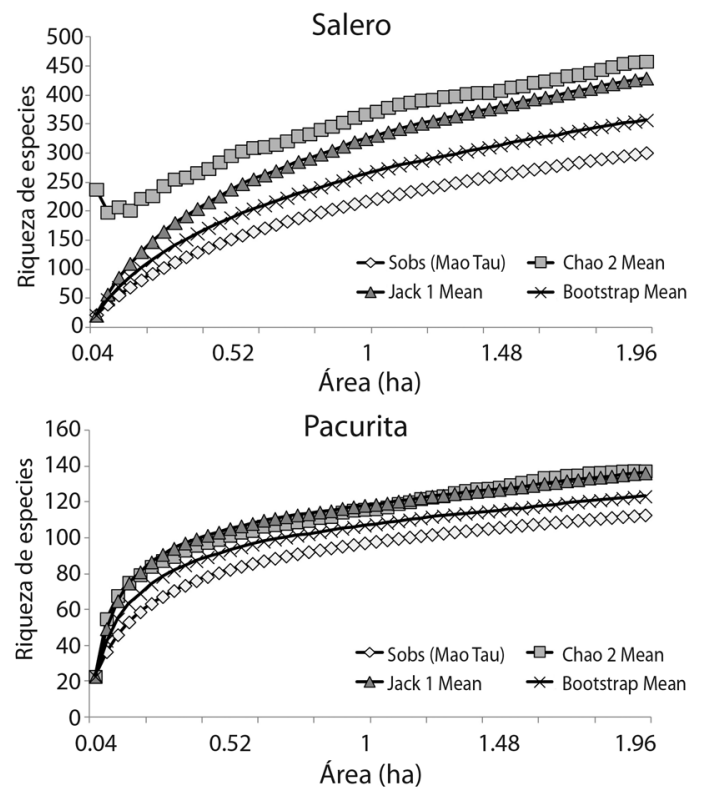

Salero los estimadores presentaron valores muy altos y los sesgos de los estimadores Jacknife 1 y Chao 2 fueron igualmente altos. Aunque en esta localidad se presentó la mayor exactitud de estimación con el Chao 2 (Tabla 2).

Mediante el coeficiente de correlación de Spearman se determinó que la riqueza y equidad arbórea se relacionaron de forma positiva con el $\mathrm{P}$ disponible, $\mathrm{Al}$, limo y arcilla; mientras que, con el pH, MO, Ca, $\mathrm{Mg}, \mathrm{K}$ y arena, la relación fue negativa (Tabla 3 ). Por su parte, las variables de dominancia se relacionaron de forma positiva con el $\mathrm{pH}, \mathrm{MO}, \mathrm{Ca}$ y arena, mientras que con el $\mathrm{P}$ y el Al la relación fue negativa (Tabla 3). El ACP presentó cuatro componentes significativos con Eigenvalue de $7.88(43.8 \%), 3.87(21.53 \%), 1.57(8.7 \%)$ y $1.15(6.39 \%)$ respectivamente que explican el $80.5 \%$ de la variación. Los dos primeros
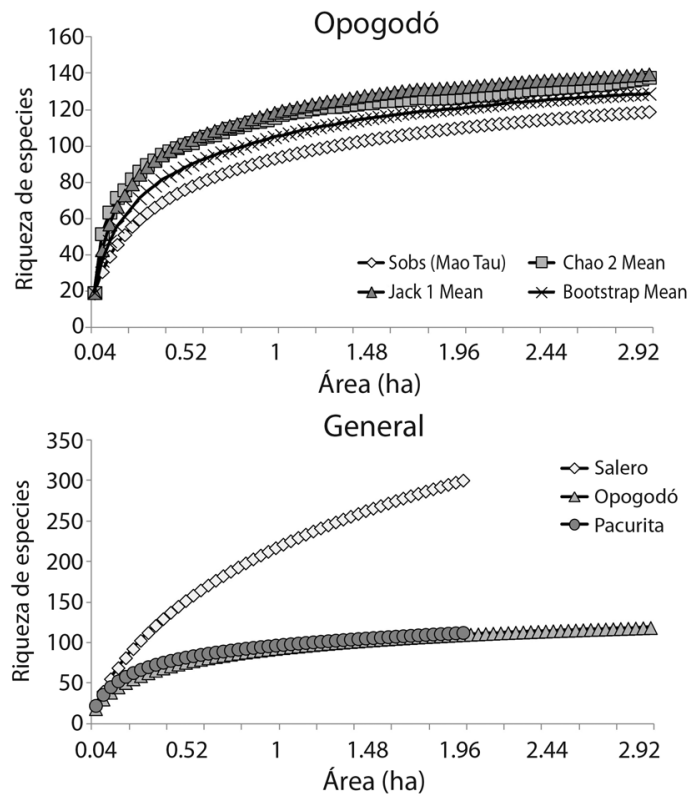

Fig. 2. Curvas de acumulación de especies de arbóreas de los bosques pluviales tropicales de Opogodó, Pacurita y Salero en el Chocó biogeográfico, Colombia.

Fig. 2. Accumulation curves of tree species from the tropical rainforests of Opogodó, Pacurita and Salero in the biogeographical Chocó, Colombia. 


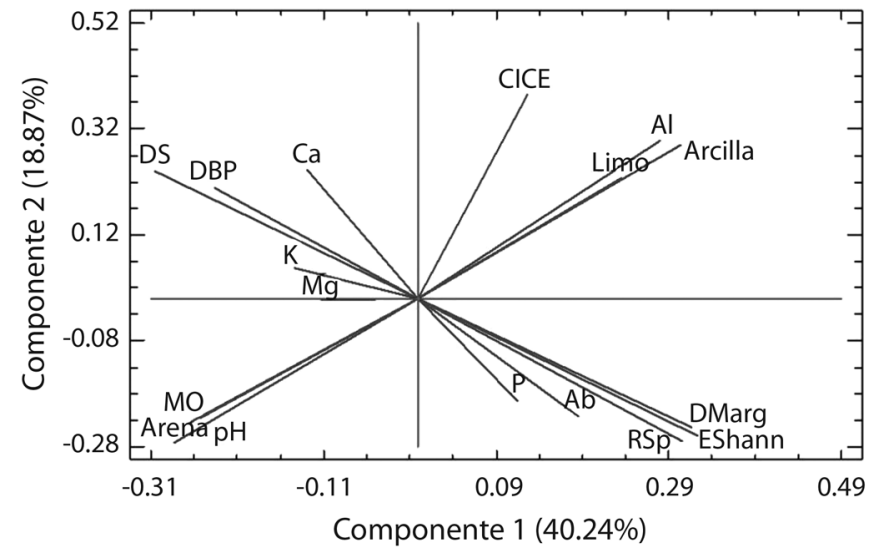

Fig. 3. Principal component analysis of tree diversity and edaphic conditions (pH, Al, MO, P, K, Ca, Mg, CICE, Clay, Limo, Sand) of the rain forests of Chocó, Colombia. In the figure, DS is the Simpson dominance index, DBP is the Berger and Parker dominance index, $\mathrm{Ab}$ is the abundance of individuals, RSp is the species richness, DMarg is the Margalef index and EShann is the index of Shannon-Weiner equity.

Fig. 3. Análisis de componentes principales de la diversidad arbórea y condiciones edáficas ( $\mathrm{pH}, \mathrm{Al}, \mathrm{MO}, \mathrm{P}, \mathrm{K}, \mathrm{Ca}, \mathrm{Mg}$, CICE, Arcilla, Limo, Arena) de los bosques pluviales del Chocó, Colombia. En la figura, DS es el índice de dominancia de Simpson, DBP es el índice de dominancia de Berger y Parker, Ab es la abundancia de individuos, RSp es la riqueza de especies, DMarg es el índice de Margalef y EShann es el índice de equidad de Shannon- Weiner.

TABLA 3

Coeficiente de correlación por rangos de Spearman para variables de diversidad arbórea y condiciones edáficas de tres bosques lluviosos tropicales (Opogodó. Pacurita y Salero) del Chocó biogeográfico, Colombia

TABLE 3

Spearman rank correlation coefficient for tree diversity variables and edaphic conditions of three tropical rainforests (Opogodó, Pacurita and Salero) of the biogeographical Chocó, Colombia

\begin{tabular}{|c|c|c|c|c|c|c|}
\hline & Riqueza & Abundancia & Shannon & Margalef & Dominancia & Berger_Parker \\
\hline $\mathrm{pH}$ & $-0.37 * *$ & $-0.38 * *$ & $-0.33 * *$ & $-0.32 * *$ & $0.27 * *$ & $0.12 \mathrm{~ns}$ \\
\hline Materia orgánica & $-0.37 * *$ & $-0.29 * *$ & $-0.35^{* *}$ & $-0.35 * *$ & $0.30 * *$ & $0.13 \mathrm{~ns}$ \\
\hline Fósforo & $0.25^{* *}$ & $0.18^{*}$ & $0.23 * *$ & $0.23 * *$ & $-0.20 * *$ & $-0.10 \mathrm{~ns}$ \\
\hline Aluminio & $0.36^{* *}$ & $0.22 * *$ & $0.38 * *$ & $0.38 * *$ & $-0.36^{* *}$ & $-0.22 * *$ \\
\hline Calcio & $-0.33 * *$ & $-0.26^{* *}$ & $-0.32 * *$ & $-0.32 * *$ & $0.29 * *$ & $0.21 * *$ \\
\hline Magnesio & $-0.16^{*}$ & $-0.25^{* *}$ & $-0.10 \mathrm{~ns}$ & $-0.10 \mathrm{~ns}$ & $0.04 \mathrm{~ns}$ & $-0.05 n s$ \\
\hline Potasio & $-0.17^{*}$ & $-0.14 n s$ & $-0.16^{*}$ & $-0.17 *$ & $0.14 \mathrm{~ns}$ & $0.05 \mathrm{~ns}$ \\
\hline CICE & $0.06 \mathrm{~ns}$ & $0.01 \mathrm{~ns}$ & $0.09 \mathrm{~ns}$ & $0.08 \mathrm{~ns}$ & $-0.10 \mathrm{~ns}$ & $-0.06 n s$ \\
\hline Arena & $-0.32 * *$ & $-0.01 \mathrm{~ns}$ & $-0.39 * *$ & $-0.41 * *$ & $0.40 * *$ & $0.26 * *$ \\
\hline Limo & $0.27 * *$ & $-0.02 \mathrm{~ns}$ & $0.34 * *$ & $0.36 * *$ & $-0.36^{* *}$ & $-0.25 * *$ \\
\hline Arcilla & $0.38 * *$ & $0.19 * *$ & $0.40 * *$ & $0.41 * *$ & $-0.37 * *$ & $-0.22 * *$ \\
\hline
\end{tabular}

$*: \mathrm{P}<0.05 ; * *: \mathrm{P}<0.01 \mathrm{~ns}:$ No significativa.

componentes que explicaron el $65.3 \%$ de la variación (Fig. 3). El ACP mostró una asociación entre la riqueza, equidad y abundancia de individuos, con el $\mathrm{P}$ disponible; así mismo, la dominancia mostró asociación con el Ca, K y Mg (Fig. 3).
Mediante la regresión lineal múltiple se determinó que la riqueza de especies y la equidad de Shannon fueron explicadas significativamente por el $\mathrm{P}$ disponible, $\mathrm{Al} \mathrm{Mg}$ y $\mathrm{CICE}$, con un porcentaje de explicación del $30.9 \%$ $\left(\mathrm{R}^{2}=0.31\right)$ para riqueza de especies, y un 32.7 
$\%\left(\mathrm{R}^{2}=0.33\right)$ para la equidad. Además, tanto la riqueza de especies y la equidad de Shannon, presentaron una relación negativa con el CICE del suelo (Tabla 4). Por su parte, la equidad mostró también una relación significativa negativa con el porcentaje de arena (Tabla 4). Finalmente, la dominancia de Simpson presentó relación significativa negativa con la $\mathrm{Al}, \mathrm{Ca}$, $\mathrm{Mg}$, y K; mientras que, con el CICE, arena y limo, la relación fue positiva, con un porcentaje de explicación del $30.4 \%\left(\mathrm{R}^{2}=0.3\right.$; Tabla 4$)$.

Además mediante una revisión de artículo se determinó que la riqueza de especies arbóreas de bosques del Chocó pueden presentar valores muy variables (bajos y altos) de diversidad arbórea (Tabla 5). Entre los cuales se puede mencionar a Gentry (1993), Galeano (2001), Mosquera, Robledo, y Asprilla (2007), Quinto y Moreno (2014), y Álvarez-Dávila et al. (2016) que presentan valores diferentes de riqueza de especies por ha (Tabla 5). Particularmente, en este estudio en Opogodó, con suelos arenosos y ricos en $\mathrm{N}$ y MO, se presentó una diversidad baja; mientras que en los suelos pobres en nutrientes y de topografía quebrada de Pacurita y Salero se presentaron valores altos de riqueza de especies.

\section{DISCUSIÓN}

Posiblemente, la alta diversidad registrada en Salero está relacionada con la forma de las parcelas. Dado que, en Salero las parcelas fueron rectangulares $(20$ x $500 \mathrm{~m})$; mientras que, en Opogodó y Pacurita, fueron cuadradas $(100 \times 100 \mathrm{~m})$. El hecho de que en Salero las parcelas fueran rectangulares probablemente permitió evaluar una mayor variedad de hábitats y ambientes edáficos (Clark, 2002), lo que seguramente contribuyó a registrar una mayor riqueza de especies arbóreas; dado que, las parcelas de Salero atraviesan mayor variedad de microhábitats, como quebradas, pendientes, áreas pantanosas y tierra firme.

La influencia de la forma de las parcelas sobre la diversidad de especies arbóreas ha sido evaluada por varios investigadores previamente (Vallejo-joyas et al., 2005). Por ejemplo el efecto de la forma de la parcela sobre la riqueza de especies del bosque evaluando tres formas: rectangulares de $20 \times 500 \mathrm{~m}$, transectos de 10 x $1000 \mathrm{~m}$ y cuadradas de 100 x $100 \mathrm{~m}$, en bosques lluviosos tropicales de Pasoh (Malasia) y Barro Colorado (Panamá); y encontraron que en las parcelas rectangulares la diversidad de especies incrementa entre 5.3-11.3\% más que en las parcelas cuadradas (Condit et al., 1996); similar a lo encontrado en este estudio, al comparar las curvas de acumulación de especies entre localidades.

La alta diversidad arbórea registrada en algunos sitios del Chocó biogeográfico, corroboran la aseveración de Gentry (1988), quien postuló a los bosques de esta región como candidatos a ser uno de los más biodiversos del Neotrópico. Particularmente, la diversidad encontrada en Salero fue similar a la encontrada en sitios de la región geográfica como Tutunendo y Bajo Calima (Clinebell et al., 1995; Gentry, 1988). En este sentido, FaberLangendoen y Gentry (1991) reportaron una riqueza de 258 especies arbóreas por hectárea en bosques del Bajo Calima, sobre suelos arcillosos, pobres en nutrientes, con muy alto contenido de Al y de topografía quebrada (Faber-Langendoen \& Gentry, 1991). Similar a lo registrado en Salero, con 219 especies arbóreas por hectárea e igualmente con suelos pobres en nutrientes, con alto contenido de $\mathrm{Al}$ y de topografía quebrada. Aunque en el Bajo Calima, las parcelas fueron cuadradas y en Salero fueron rectangulares. Asimismo, Galeano (2001), registró 294 especies arbóreas en 1.8 ha en bosques del Golfo de Tribugá (Chocó biogeográfico) con suelos arenosos y ricos en nutrientes.

En este estudio se observó que la riqueza y equidad de especies presentan una correlación negativa con algunas variables de fertilidad del suelo, específicamente con nutrientes del suelo como $\mathrm{Ca}, \mathrm{Mg}, \mathrm{K}$ y MO. Estos resultados son similares a los reportados por Huston (1980) quien evidenció una relación negativa entre la disponibilidad de nutrientes $(\mathrm{P}, \mathrm{K}, \mathrm{Ca}, \mathrm{Na}$, bases totales y CICE) del suelo y la riqueza de especies en bosques lluviosos de Costa Rica. 
TABLA 4

Regresiones lineales múltiples de las variables de diversidad arbórea y condiciones edáficas de los bosques pluviales tropicales (Opogodó. Pacurita y Salero) del Chocó biogeográfico, Colombia. El método de Stepwise - backward

TABLE 4

Multiple linear regressions of tree diversity variables and edaphic conditions of the tropical rain forests (Opogodó, Pacurita and Salero) of the biogeographical Chocó, Colombia. The Stepwise-backward method

\begin{tabular}{lcccc}
\multicolumn{3}{c}{ Riqueza de especies } \\
Parámetro & Estimación & Error Estándar & Estadístico T & Valor-P \\
CONSTANTE & 21.2777 & 2.47234 & 8.60632 & 0.0000 \\
LOG(Fósforo+1) & 4.65855 & 1.48164 & 3.14418 & 0.0020 \\
LOG(Aluminio+1) & 16.5579 & 2.73334 & 6.05775 & 0.0000 \\
LOG(Magnesio+1) & 12.3497 & 4.66154 & 2.64927 & 0.0089 \\
LOG(CICE+1) & -15.4497 & 3.66902 & -4.21084 & 0.0000 \\
\hline
\end{tabular}

\begin{tabular}{lccccc} 
& \multicolumn{5}{c}{ Análisis de Varianza } \\
\multicolumn{1}{c}{ Fuente } & Suma de Cuadrados & Gl & Cuadrado Medio & Razón-F & Valor-P \\
Modelo & 1112.56 & 4 & 278.14 & 16.62 & 0.0000 \\
Residuo & 2476.5 & 148 & 16.7331 & & \\
Total (Corr.) & 3589.06 & 152 & & & \\
\hline
\end{tabular}

$\mathrm{R}$-cuadrada $=30.9986$ porciento.

\begin{tabular}{lcccc}
\multicolumn{3}{c}{ Equidad de Shannon } \\
Parámetro & Estimación & Error Estándar & Estadístico T & Valor-P \\
CONSTANTE & 3.4913 & 0.298863 & 11.6819 & 0.0000 \\
LOG(Fósforo+1) & 0.185171 & 0.073307 & 2.52596 & 0.0126 \\
LOG(Aluminio+1) & 0.69785 & 0.145877 & 4.78383 & 0.0000 \\
LOG(Magnesio+1) & 0.590536 & 0.228008 & 2.58998 & 0.0106 \\
LOG(CICE+1) & -0.723614 & 0.179655 & -4.0278 & 0.0001 \\
LOG(Arena+1) & -0.131039 & 0.0605776 & -2.16316 & 0.0321 \\
\hline
\end{tabular}

\begin{tabular}{lccccc} 
& \multicolumn{5}{c}{ Análisis de Varianza } \\
\multicolumn{1}{c}{ Fuente } & Suma de Cuadrados & Gl & Cuadrado Medio & Razón-F & Valor-P \\
Modelo & 2.85909 & 5 & 0.571817 & 14.29 & 0.0000 \\
Residuo & 5.88327 & 147 & 0.0400222 & & \\
Total (Corr.) & 8.74236 & 152 & & & \\
\hline
\end{tabular}

$\mathrm{R}$-cuadrada $=32.7038$ porciento.

\begin{tabular}{lcccc}
\multicolumn{1}{c}{ Parámetro } & Estimación & Error Estándar & Estadístico T & Valor-P \\
CONSTANTE & -0.14128 & 0.0619603 & -2.28017 & 0.0241 \\
LOG(Aluminio+1) & -0.175069 & 0.0603715 & -2.89986 & 0.0043 \\
LOG(Calcio+1) & -0.114681 & 0.0559885 & -2.0483 & 0.0423 \\
LOG(Magnesio+1) & -0.153896 & 0.0569567 & -2.70198 & 0.0077 \\
LOG(Potasio+1) & -0.108556 & 0.0508738 & -2.13384 & 0.0345 \\
LOG(CICE+1) & 0.235662 & 0.0867237 & 2.71739 & 0.0074 \\
LOG(Arena+1) & 0.0298516 & 0.0107121 & 2.78671 & 0.0060 \\
LOG(Limo+1) & 0.0138098 & 0.00623789 & 2.21386 & 0.0284 \\
\hline
\end{tabular}

\begin{tabular}{lccccc} 
& \multicolumn{2}{c}{ Análisis de Varianza } \\
\multicolumn{1}{c}{ Fuente } & Suma de Cuadrados & Gl & Cuadrado Medio & Razón-F & Valor-P \\
Modelo & 0.0159746 & 7 & 0.00228208 & 9.06 & 0.0000 \\
Residuo & 0.0365327 & 145 & 0.000251949 & & \\
Total (Corr.) & 0.0525072 & 152 & & & \\
\hline
\end{tabular}

R-cuadrada $=30.42$ por ciento. 
TABLA 5

Diversidad y abundancia de especies arbóreas de bosques lluviosos tropicales del Chocó biogeográfico, Colombia

TABLE 5

Diversity and abundance of tree species from tropical rainforests of the biogeographical Chocó, Colombia

\begin{tabular}{lcccccl}
\multicolumn{1}{c}{ Sitios } & $\begin{array}{c}\mathrm{N}^{\circ} \\
\text { Individuos }\end{array}$ & $\begin{array}{c}\mathrm{N}^{\circ} \text { de } \\
\text { Especies }\end{array}$ & Margalef & $\begin{array}{c}\text { Área } \\
(\mathrm{ha})\end{array}$ & $\begin{array}{c}\text { DAP } \\
(\mathrm{cm})\end{array}$ & \multicolumn{1}{c}{ Referencia } \\
Tutunendo 1. Colombia & 763 & 103 & 15.37 & 1.0 & $\geq 10$ & Citado por Mosquera et al. (2007) \\
Tutunendo 2. Colombia & 717 & 92 & 13.84 & 1.0 & $\geq 10$ & Citado por Mosquera et al. (2007) \\
Pacurita. Colombia & 158 & 66 & 12.84 & 0.3 & $\geq 10$ & García et al., (2004) \\
San Martin. Colombia & 112 & 49 & 10.17 & 0.3 & $\geq 10$ & García et al., (2004) \\
San José. Colombia & 298 & 124 & 21.59 & 0.3 & $\geq 10$ & García et al., (2004) \\
Guadalupe. Colombia & 192 & 44 & 8.18 & 0.3 & $\geq 10$ & García et al., (2004) \\
Pacurita. Colombia & 654 & 95 & 14.50 & 1.0 & $\geq 10$ & Citado por Mosquera et al., (2007) \\
Coquí. Colombia & 471 & 158 & 25.51 & 0.4 & $\geq 5$ & Galeano (2001) \\
Nuquí. Colombia & 414 & 151 & 24.89 & 0.4 & $\geq 5$ & Galeano (2001) \\
Bajo Calima. Colombia & 675 & 258 & 39.45 & 1.0 & $\geq 10$ & Faber-Langendoen y Gentry (1991) \\
Opogodó. Colombia & 955 & 90 & 12.97 & 1.0 & $\geq 10$ & Este estudio \\
Opogodó. Colombia & 708 & 75 & 11.28 & 1.0 & $\geq 10$ & Este estudio \\
Opogodó. Colombia & 636 & 78 & 11.93 & 1.0 & $\geq 10$ & Este estudio \\
Pacurita. Colombia & 913 & 97 & 14.08 & 1.0 & $\geq 10$ & Este estudio \\
Pacurita. Colombia & 979 & 93 & 13.36 & 1.0 & $\geq 10$ & Este estudio \\
Salero. Colombia & 712 & 219 & 33.19 & 1.0 & $\geq 10$ & Este estudio \\
Salero. Colombia & 644 & 179 & 27.52 & 1.0 & $\geq 10$ & Este estudio \\
\hline
\end{tabular}

Asimismo, Clinebell et al., (1995) reportó que la riqueza se relaciona negativamente con la disponibilidad de nutrientes $(\mathrm{K}, \mathrm{Ca}, \mathrm{Mg}$ y $\mathrm{P}$ ) en un meta-análisis a nivel de bosques Neotropicales. Basados en lo anterior, Huston $(1980,2012)$ consideró que la mayor riqueza ocurre bajo condiciones que restringen el crecimiento arbóreo.

Por otra parte, la relación entre algunos nutrientes como $\mathrm{P}$ disponible y $\mathrm{Mg}$ con la diversidad y equidad arbórea fue positiva, estas fueron las variables edáficas que mejor explicaron la riqueza y equidad. Esto se relaciona con lo encontrado por Condit et al., (2013) que evidenciaron una asociación entre la composición y riqueza de especies arbóreas y el contenido de humedad de la estación seca, el $\mathrm{P}$ disponible y el $\mathrm{Ca}$. Al parecer, bajo condiciones de baja fertilidad edáfica, la concentración de $\mathrm{P}$ disponible puede tener un efecto significativo y positivo sobre la diversidad arbórea.

Huston (1980) y Clinebell et al., (1995) encontraron que la riqueza de especies presenta correlaciones positivas con la precipitación, pero negativa con la fertilidad del suelo. Así mismo, la fertilidad del suelo presenta correlación negativa con la precipitación (Clinebell et al., 1995; Huston, 1980), lo cual es consistente con que en condiciones de alta precipitación y baja fertilidad edáfica la riqueza de especies tiende a ser mayor (Huston, 2012). Sin embargo, Gentry $(1982,1988)$ y Clinebell et al. (1995) consideraron que la precipitación anual y los meses de sequias son las variables que mejor explican la riqueza de especies y no las variables edáficas como disponibilidad de nutrientes, contrario a lo planteado por Huston (1980, 2012). Particularmente, los estudios realizados en bosques del Chocó muestran que las condiciones edáficas explican poco la diversidad arbórea, como se evidenció en Quinto y Moreno (2014) y en el presente estudio; los nutrientes aunque muestran correlaciones significativas con las variables de diversidad arbórea su porcentaje de explicación es muy bajo $(<40 \%)$. La posible razón de este bajo 
porcentaje de explicación puede estar relacionada con la influencia de otras variables ambientales y biológicas como la limitación en dispersión, clima, entre otros (Duque et al., 2003; Valencia et al., 2004), que al parecer están teniendo mayor influencia sobre la diversidad arbórea.

Aunque una correlación no necesariamente representa causalidad, la pregunta que surge es: ¿por qué bajo condiciones de alta precipitación y baja disponibilidad de nutrientes, la riqueza de especies arbóreas tiende a ser alta en bosques tropicales? Según Huston (1980) la alta precipitación genera reducción en los niveles de nutrientes del suelo por lixiviación (Austin \& Vitousek, 1998; Quinto \& Moreno, 2016; Santiago, Schuur, \& Silvera, 2005); lo cual, produce una mayor limitación del crecimiento arbóreo (Lambers, Chapin III, Pons, \& Thijs, 2008; Marschner, 1991; Salisbury \& Ross, 1994), pero conduce a una mayor abundancia de árboles pequeños. La alta precipitación no solo reduce la cantidad de nutrientes por lixiviación, sino que también produce mayor acidez y concentración edáfica de Al (Jenny, 1941; Austin \& Vitousek, 1998). Por consiguiente, con alta precipitación tienden a presentarse altos contenidos de $\mathrm{Al}$ edáfico que reducen las tasas de crecimiento arbóreo (Marschner, 1991) y ocasiona una mayor riqueza de especies (Huston, 1980). Esto explicaría porque se encontró una relación positiva entre la riqueza de especies arbóreas y el contenido de $\mathrm{Al}$ edáfico en bosques del Chocó biogeográfico (Quinto \& Moreno, 2014) y en otros bosques lluviosos tropicales (Clinebell et al., 1995). Asimismo, investigaciones realizadas en el Chocó y la Amazonia han mostrado una alta diversidad arbórea sobre suelos con alto contenido de $\mathrm{Al}$ (Duivenvoorden, 1996; Faber-Langendoen \& Gentry, 1991).

Phillips et al., (1994) concluyeron que los bosques lluviosos tropicales con mayor dinámica (mortalidad y reclutamiento) tienden a presentar alta riqueza de especies. Asimismo, los pequeños disturbios (claros naturales) ayudan a mantener la diversidad. Probablemente, factores como la toxicidad por Al, topografía quebrada, acidez del suelo y baja disponibilidad de nutrientes, podrían generar mayor dinámica del bosque (Asquith, 2002; Laurance et al., 2010; Marschner, 1991); y por ende, una mayor diversidad arbórea (Phillips et al., 1994). Posiblemente esta interacción de factores es la que mejor explica porque se presenta una alta diversidad arbórea en bosques tropicales de alta precipitación, con suelos infértiles y con alta toxicidad por Al (Clinebell et al., 1995; Duivenvoorden, 1996). En síntesis, la alta diversidad arbórea registrada en estos bosques del Chocó está determinada considerablemente por el contenido edáfico de nutrientes. Asimismo, la baja fertilidad edáfica, acidez y toxicidad por $\mathrm{Al}$, generados por la precipitación, están determinando la dinámica y la diversidad arbórea.

Declaración de ética: los autores declaran que todos están de acuerdo con esta publicación y que han hecho aportes que justifican su autoría; que no hay conflicto de interés de ningún tipo; y que han cumplido con todos los requisitos y procedimientos éticos y legales pertinentes. Todas las fuentes de financiamiento se detallan plena y claramente en la sección de agradecimientos. El respectivo documento legal firmado se encuentra en los archivos de la revista.

\section{RESUMEN}

Introducción: El Chocó biogeográfico posee una exuberante diversidad de especies arbóreas; no obstante, se desconoce que tanto los suelos determinan dicha diversidad. Objetivo: Se evaluó el efecto de las condiciones edáficas sobre la diversidad y dominancia arbórea en bosques lluviosos tropicales de la región. Métodos: Se establecieron siete parcelas permanentes de investigación en las localidades de Opogodó, Pacurita y Salero. En dichas parcelas, se midió la riqueza, equidad y dominancia arbórea, además se determinaron las condiciones edáficas como MO, acidez, nutrientes y textura. Resultados: Se encontró, que la diversidad fue mayor en Salero y Pacurita; pero la dominancia fue mayor en Opogodó. La diversidad se relacionó positivamente con el $\mathrm{P}$ disponible, $\mathrm{Al}$ intercambiable, porcentajes de limo y arcilla; mientras que, con el $\mathrm{pH}, \mathrm{MO}$, basas intercambiables de $\mathrm{Ca}, \mathrm{Mg}, \mathrm{K}$ y porcentaje de arena, la relación fue negativa. Por su parte, la dominancia se relacionó negativamente con el $\mathrm{P}$ y el Al; mientras que, la relación fue positiva con el pH, MO, Ca 
y porcentaje de arena. Conclusión: Se corroboró que la diversidad arbórea se relaciona negativamente con algunas variables $(\mathrm{Ca}, \mathrm{Mg}, \mathrm{K})$ de fertilidad edáfica. En síntesis, la infertilidad edáfica, acidez y toxicidad por Al, determinan la diversidad y dominancia arbórea.

Palabras clave: aluminio, fertilidad edáfica, fósforo, Opogodó, Pacurita, Salero, diversidad, dominancia arbórea.

\section{REFERENCIAS}

Álvarez-Dávila, E., Jaramillo-Giraldo, G. C., CogolloRivera, C. C., Martínez-Higuera, H., Rojas, E., \& Fernández-Méndez, F. (2016). Structure and diversity of the three plant associations in the San Juan River Delta, Chocó, Colombia. Revista Árvore, 40(5), 833-843.

Antonelli, A., \& Sanmartin, I. (2011). Why are there so many plant species in the Neotropics? Taxon, 60(2), 403-414.

Asquith, N. (2002). La Dinámica del Bosque y la Diversidad Arbórea. En M. R. Guariguata, \& G. H. Kattan (Eds.), Ecología y Conservación de Bosques Neotropicales (pp. 377-406). Costa Rica: Libro Universitario Regional.

Austin, A. T., \& Vitousek, P. M. (1998). Nutrient dynamics on a precipitation gradient in Hawaii. Oecologia, 113(4), 519-529.

Barreto, J. S., Duque, A., Cárdenas, D., \& Moreno, F. (2010). Variación florística de especies arbóreas a escala local en un bosque de tierra firme en la Amazonia colombiana. Acta Amazónica, 40, 179-188.

Chao, A., Chazdon, R. L., Colwell, R. K., \& Shen, T. J. (2005). A new statistical approach for assessing compositional similarity based on incidence and abundance data. Ecology Letter, 8, 148-159.

Chiarucci, A., Enright, N. J., Perry, G. L., Miller, B. P., \& Lamont, B. B. (2003). Performance of nonparametric species richness estimators in a high diversity plant community. Diversity and Distributions, 9, 283-295.

Clark, D. B. (2002). Factores edáficos y la distribución de plantas a nivel del paisaje en bosques húmedos neotropicales. In M. Guariguata, \& G. Kattan (Eds.), Ecología de bosques lluviosos neotropicales. Costa Rica: Instituto Interamericano de Ciencias Agrícolas.

Clark, D. B., Clark, D. A., \& Read, J. M. (1998). Edaphic variation and the mesoscale distribution of tree species in a neotropical rain forest. Journal of Ecology, $86,101-112$.

Clinebell, R. R., Phillips, O. L., Gentry, A. H., Stark, N., \& Zuuring, H. (1995). Prediction of neotropical tree and liana species richness from soil and climatic data. Biodiversity and Conservation, 4, 56-90.
Colwell, R. K., Mao, C. X., \& Chang, J. (2004). Interpolating, extrapolating, and comparing incidencebased species accumulation curves. Ecology, 85, 2717-2727.

Colwell, R. K., Chao, A., Gotelli, N. J., Lin, S. Y., Mao, C. X., Chazdon, R. L., \& Longino, J. T. (2012). Models and estimators linking individual-based and samplebased rarefaction, extrapolation, and comparison of assemblages. Journal of Plant Ecology, 5, 3-21.

Condit, R., Engelbrecht, B. M. J., Pino, D., Pérez, R., \& Turner, B. L. (2013). Species distributions in response to individual soil nutrients and seasonal drought across a community of tropical trees. Proceedings of the National Academy of Sciences USA, 110(13), 5064-5068.

Condit, R., Hubbell, S. P., LaFrankie, J. V., Sukumar, R., Manokaran, N., Foster, R. B., \& Ashton, P. S. (1996). Species-area and species-individual relationships for tropical trees: a comparison of three 50-ha plots. Journal of Ecology, 84, 549-562.

Duivenvoorden, J. F. (1996). Patterns of tree species richness in rain forest of the middle Caquetá area, Colombia, NW, Amazonia. Biotropica, 28, 142-158.

Duque, A., Cavelier, J., \& Posada, A. (2003). Strategies of tree occupation at a local scale in terra firme Forests in the Colombian Amazon. Biotropica, 35, 20-27.

Faber-Langendoen, D., \& Gentry, A. H. (1991). The structure and diversity of rain forests at Bajo Calima, Chocó Region, Western Colombia. Biotropica, 23(1), $2-11$.

Galeano, G. (2001). Estructura, riqueza y composición de plantas leñosas en el golfo de Tribugá, Chocó, Colombia. Caldasia, 23(1), 213-236.

García, F., Ramos, Y., Palacios, J., Arroyo, J. E., Mena, A., \& González, M. (2003). Salero: Diversidad Biológica de un Bosque Pluvial Tropical (bp-T). Colombia: Editorial Guadalupe Ltda.

García, F., Moreno, M., Robledo, D., Mosquera, L., \& Palacios, L. (2004). Composición y diversidad florística de los bosques de la cuenca hidrográfica del río Cabí, Quibdó-Chocó. Revista Institucional Universidad Tecnológica del Chocó, 20(1), 13-23.

Gentry, A. (1988). Changes in Plant Community Diversity and Floristic Composition on Environmental and Geographical Gradients. Annals of the Missouri Botanical Garden, 75(1), 1-34.

Gentry, A. (1993). A field guide to the families and genera of woody plants of Northwest South Amercian (Colombia, Ecuador, and Peru). Washington, DC. USA.: Conservation International.

Gentry, A. H. (1982). Neotropical floristic diversity: phytogeographical connections between Central and South 
America, Pleistocene climatic fluctuations, or an accident of the Andean orogeny? Annals of the Missouri Botanical Garden, 69, 557-593.

Givnish, T. J. (1999). On the Causes of Gradients in Tropical Tree Diversity. The Journal of Ecology, 87(2), 193-210.

Hammer, Ø., Harper, D. A. T., \& Ryan, P. D. (2001). PAST: Paleontological statistics software package for education and data analysis. Palaeontologia Electronica, $4(1), 1-9$

Hartshorn, G. S. (2002). Biogeografía de los bosques neotropicales. En M. R. Guariguata, \& G. H. Kattan (Eds.), Ecología y conservación de bosques neotropicales (pp. 59-81). Costa Rica: LUR.

Hill, M. O. (1973). Diversity and evenness: a unifying notation and its consequences. Ecology, 54(2), 427-432.

Hoshmand, A. R. (1998). Statistical Methods for Environmental \& Agricultural Sciences (Segunda Edición). U.S.A.: CRC Press LLC.

Huston, M. A. (1980). Soil nutrients and tree species richness in Costa Rican forests. Journal of Biogeography, 7(1), 147-157.

Huston, M. A. (1994). Biological diversity: the coexistence of species on changing landscapes. U.S.A.: Cambridge University Press.

Huston, M. A. (2012). Precipitation, soils, NPP, and biodiversity: resurrection of Albrecht's curve. Ecological Monographs, 82(3), 277-296.

Jenny, H. (1941). Factors of soil formation. U.S.A.: McGraw-Hill.

Jost, L. (2006). Entropy and diversity. Oikos, 113(2), 363-375.

Lambers, H., Chapin III, F., Pons, S., \& Thijs, L. (2008) Plant Physiological Ecology (2 ${ }^{\text {nd }}$ Edition). U.S.A.: Springer-Verlag.

Laurance, S. G. W., Laurance, W. F., Andrade, A., Fearnside, P. M., Harms, K. E., Vicentini, A., \& Luizão, R. C. C. (2010). Influence of soils and topography on Amazonian tree diversity: a landscape-scale study. Journal of Vegetation Science, 21, 96-106.

Leigh, E. G., Davidar, P., Dick, C. W., Puyravaud, J. P., Terborgh, J., Ter Steege, H., \& Wright, S. J. (2004). Why do some tropical forests have so many species of trees? Biotropica, 36, 447-473.

López-Gómez, A. M., \& Williams-Linera, G. (2006) Evaluación de métodos no paramétricos para la estimación de riqueza de especies de plantas leñosas en cafetales. Boletín de la Sociedad Botánica de México, 78(1), 7-15.
Magurran, A. E. (1988). Ecological diversity and its measurement. U.S.A.: Princeton University Press.

Mahecha, G. (1997). Fundamentos y metodologías para la identificación de plantas. Colombia: Ministerio del Medio Ambiente, GEF - PNUD. Lerner Ltda.

Marschner, H. (1991). Mechanisms of adaptation of plants to acid soils. Plant and Soil, 134(1), 1-20.

Moreno, C. E. (2001). Métodos para medir la biodiversidad (Volumen 1). España: M\&T-Manuales y Tesis SEA.

Mosquera, L. J., Robledo, D., \& Asprilla, A. (2007). Diversidad florística de dos zonas de bosque tropical húmedo en el municipio de Alto Baudó, Chocó, Colombia. Revista Acta Biológica Colombiana, 12, 75-90.

Myers, N., Mittermeier, R. A., Mittermeier, C. G., Da Fonseca, G. A., \& Kent, J. (2000). Biodiversity hotspots for conservation priorities. Nature, 403, 853-858.

Nadeau, M. B. \& Sullivan, T. P. (2015). Relationships between Plant Biodiversity and Soil Fertility in a Mature Tropical Forest, Costa Rica. International Journal of Forestry Research, 2015, 1-15.

Phillips, O. L., Hall, R., Gentry, A. R., Sawyer, S. A., \& Vizquez, R. (1994). Dynamics and species richness of tropical forests. Proceedings of the National Academy of Sciences. USA, 91, 2805-2809.

Quinto, H., \& Moreno, F. (2014). Diversidad florística arbórea y su relación con el suelo en un bosque pluvial tropical del Chocó biogeográfico. Revista Arvore, 38(6), 1123-1132.

Quinto, H., \& Moreno, F. H. (2016). Precipitation effects on soil characteristics in tropical rain forests of the Chocó biogeographical region. Revista Facultad Nacional de Agronomía Medellín, 69(1), 7813-7823.

Salisbury, F. B., \& Ross, C. W. (1994). Fisiología Vegetal (4 ${ }^{\text {ta }}$ Edición). México: Grupo Editorial Iberoamérica S.A.

Santiago, L. S., Schuur, E. A. G., \& Silvera, K. (2005). Nutrient cycling and plant-soil feedbacks along a precipitation gradient in lowland Panamá. Journal of Tropical Ecology, 21(4), 461-470.

Statistical Graphics Corporation. (2002). Statgraphics Centuriun $X V$ (Software). Recuperado de http://www. statgraphics.com

ter Braak, C. J. F. \& Šmilauer, P. (2002). CANOCO Reference Manual and CanoDraw for Windows User's Guide: Software for Canonical Community Ordination (Software, version 4.5). Ithaca NY, USA: Microcomputer Power. 
Valencia, R., Balslev, H., Paz, H., \& Mino, C. G. (1994). High tree alpha-diversity in Amazonian Ecuador. Biodiversity and Conservation, 3, 21-28.

Valencia, R., Foster, R. B., Villa, G., Condit, R., Svenning, J. C., Hernandez, C., Romoleroux, K., Losos, E., Magard, E., \& Balslev, H. (2004). Tree species distributions and local habitat variation in the Amazon: large forest plot in eastern Ecuador. Journal of Ecology, 92, 214-229.
Vallejo-Joyas, M. I., Londoño-Vega, A. C. López-Camacho, R., Galeano, G., Álvarez-Dávila, E., \& DeviaÁlvarez, W. (2005). Establecimiento de parcelas permanentes en bosques de Colombia. Colombia: Instituto de Investigación de Recursos Biológicos Alexander von Humboldt.

Wright, S. J. (2002). Plant diversity in tropical forests: a review of mechanisms of species coexistence. Oecologia, 130, 1-14. 